\title{
Coal and Coal Constituent Studies by Advanced EMR Techniques
}

\author{
Semi-Annual Report \\ September 1, 1997 - February 28, 1998
}

\author{
By \\ R. Linn Belford; Robert B. Clarkson \\ Alex I. Smirnov; Mark J. Nilges
}

Work Performed Under Contract No.: DE-FG22-96PC96205

\author{
For \\ U.S. Department of Energy \\ Office of Fossil Energy \\ Federal Energy Technology Center \\ P.O. Box 880 \\ Morgantown, West Virginia 26507-0880 \\ By \\ University of Illinois at Urbana-Champaign \\ Department of Chemistry \\ 600 S. Mathews \\ Urbana, Illinois 61801
}




\section{Disclaimer}

This report was prepared as an account of work sponsored by an agency of the United States Government. Neither the United States Government nor any agency thereof, nor any of their employees, makes any warranty, express or implied, or assumes any legal liability or responsibility for the accuracy, completeness, or usefulness of any information, apparatus, product, or process disclosed, or represents that its use would not infringe privately owned rights. Reference herein to any specific commercial product, process, or service by trade

name, trademark, manufacturer, or otherwise does not necessarily constitute or imply its endorsement, recommendation, or favoring by the United States Government or any agency thereof. The views and opinions of authors expressed herein do not necessarily state or reflect those of the United States Government or any agency thereof. 


\title{
COAL AND COAL CONSTITUENT STUDIES BY ADVANCED EMR TECHNIQUES
}

\author{
Semi-Annual Report \\ Reporting Period Start Date: 09/01/97 End Date: 02/28/98 \\ Report Issue Date: 03/31/98 \\ DE-FG22-96PC96205--03 \\ Authors: \\ R. Linn Belford \\ Robert B. Clarkson \\ Alex I. Smirnov \\ Mark J. Nilges \\ University of Illinois at Urbana-Champaign \\ Depart of Chemistry \\ 600 S. Mathews \\ Urbana, IL 61801
}


Semiannual Technical Report to DOE - PETC - Grant \# DE-FG22-96PC96205 9/01/97-2/28/98

COAL AND COAL CONSTITUENTS STUDIES BY ADVANCED EMR TECHNIQUES

\author{
by R. Linn Belford, Robert B Clarkson, Alex I. Smirnov, and Mark J. Nilges
}

University of Illinois at Urbana-Champaign

\title{
DISCLAIMER
}

This report was prepared as an account of work sponsored by an agency of the United States Government. Neither the United States Government nor any agency thereof, nor any of their employees, makes any warranty, express or implied, or assumes any legal liability or responsibility for the accuracy, completeness, or usefulness of any information, apparatus, product, or process disclosed, or represents that its use would not infringe privately owned rights. Reference herein to any specific commercial product, process, or service by trade name, trademark, manufacturer, or otherwise does not necessarily constitute or imply its endorsement, recommendation, or favoring by the United States Government or any agency thereof. The views and opinions of authors expressed herein do not necessarily state or reflect those of the United States Government or any agency thereof.

\begin{abstract}
Advanced electronic magnetic resonance (EMR) methods are used to examine properties of coals, chars, and molecular species related to constituents of coal. We have achieved substantial progress on upgrading the high field (HF) EMR (W-band, $95 \mathrm{GHz}$ ) spectrometers that are especially advantageous for such studies. Particularly, we have built a new second W-band instrument (Mark II) in addition to our Mark I. Briefly, Mark II features: (i) an Oxford custombuilt $7 \mathrm{~T}$ superconducting magnet which is scannable from 0 to $7 \mathrm{~T}$ at up to $0.5 \mathrm{~T} / \mathrm{min}$; (ii) watercooled coaxial solenoid with up to $\pm 550 \mathrm{G}$ scan under digital (15 bits resolution) computer control; (iii) custom-engineered precision feed-back circuit, which is used to drive this solenoid, is based on an Ultrastab 860R sensor that has linearity better than $5 \mathrm{ppm}$ and resolution of 0.05 ppm; (iv) an Oxford CF 1200 cryostat for variable temperature studies from 1.8 to $340 \mathrm{~K}$. During this grant period we have completed several key upgrades of both Mark I and II, particularly microwave bridge, W-band probehead, and computer interfaces. We utilize these improved instruments for HF EMR studies of spin-spin interaction and existence of different paramagnetic species in carbonaceous solids.
\end{abstract}

Page 2 of 
Semiannual Technical Report to DOE - PETC - Grant \# DE-FG22-96PC96205 9/01/97-2/28/98

\section{TABLE OF CONTENTS}

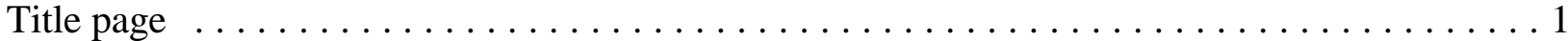

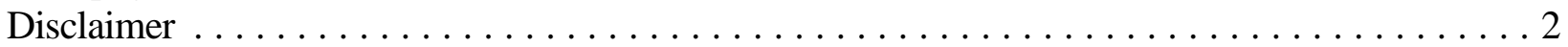

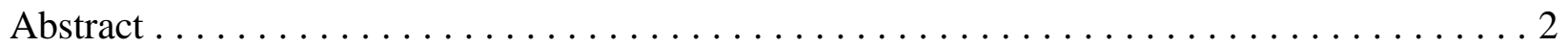

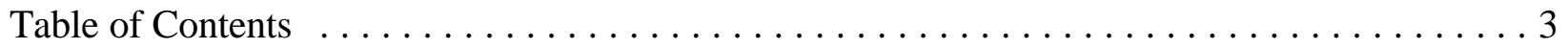

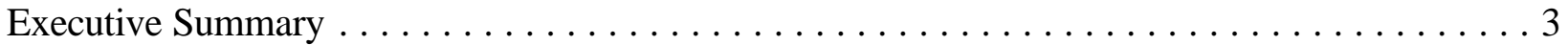

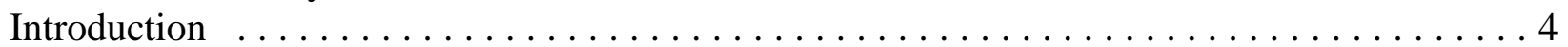

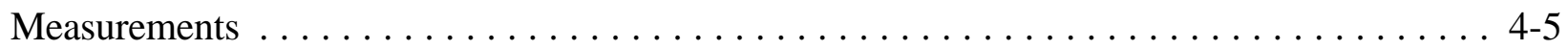

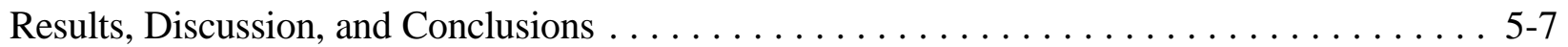

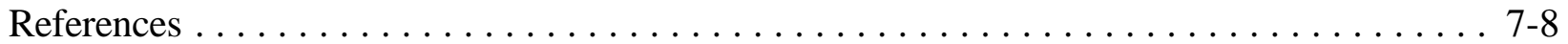

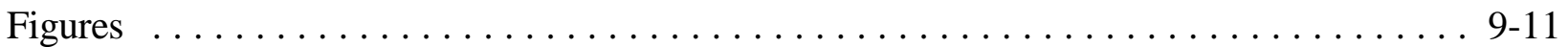

1. Experimental $9.5 \mathrm{GHz}$ (X-band) EMR spectrum of deoxygenated fusinite sample and the results of least-squares simulations based on high-temperature-limit dipolar model $(\mathrm{A}) .(\mathrm{B})$ is the fit residual. . . . . . . . . . . . . . . . . . . . . . . 9

2. Kinetics of apparent peak-to-peak line width of the fusinite sample in course of

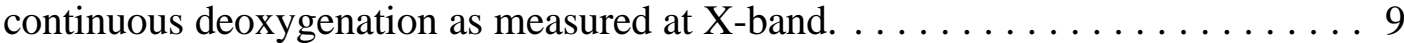

3. Gradual changes in $95 \mathrm{GHz}$ EMR spectra of fusinite in course of deoxygenation. 10

4. Experimental $95 \mathrm{GHz}$ (X-band) EMR spectrum of fusinite sample deoxygenated for $16 \mathrm{hrs}$ superimposed on the results of least-squares simulations; (B) is the simulation of the first component; (C) is the second component; (D) is the fit

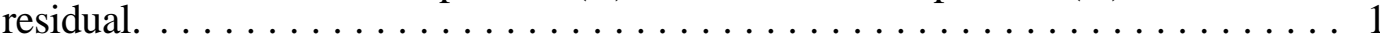

\section{EXECUTIVE SUMMARY}

The main advanced magnetic resonance method that we report for the third half-year of this project was W-band (95 GHz) electron magnetic resonance (EMR, EPR, or ESR) spectroscopy. High field (HF) EMR, which usually refers to EMR at $>90 \mathrm{GHz}$, among other specific advantages, provides an analytical tool to discriminate among very similar paramagnetic species that are constituents of coals and closely related materials such as chars. These species are difficult to identify from conventional EMR (X-band, $9.5 \mathrm{GHz}$ ) because of insufficient spectral resolution for radicals with similar g-factors. In addition to that conditions for fast Heisenberg exchange are often fulfilled at this frequency resulting in a featureless single-line EMR spectrum at 9.5 GHz. At $95 \mathrm{GHz}$ (W-band), the g-factor resolution is ten-fold of that at X-band and the frequency of experiment usually exceeds the exchange frequency. This yields an information-rich spectrum. This report describes some experimental results obtained at $95 \mathrm{GHz}$ and also considerable progress made on enhancing our W-band spectrometers used in such studies.

This report focuses on the HF EMR work. The previous two Technical Reports described the use of pulsed proton DNP to study liquid-solid interactions in aqueous slurries of several newly synthesized chars. This report focuses primarily on studies of co-existing paramagnetic species and spin-spin interactions between those in carbonaceous materials and the role of molecular oxygen as a possible "bridge" for those magnetic interactions. 
Semiannual Technical Report to DOE - PETC - Grant \# DE-FG22-96PC96205 9/01/97-2/28/98

Page 4 of 
Semiannual Technical Report to DOE - PETC - Grant \# DE-FG22-96PC96205 9/01/97-2/28/98

\section{INTRODUCTION}

The first Illinois $95 \mathrm{GHz}$ (W-band) EPR spectrometer (Mark I) was designed and built as a technological research and development project under the NIH Research Resources program and became operational in 1988-9. At that time, it was one of only two $95 \mathrm{GHz}$ spectrometers in the world. Briefly, Mark I has a heterodyne bridge design with reference arm and is equipped with a narrow bore Varian XL-200 superconducting magnet operating in persistent mode at $3.3608 \mathrm{~T}$ and an air-cooled scanning coil of $300 \mathrm{G}$ range [1]. Using this spectrometer, we have observed variety of EMR line shapes for different coal samples and different macerals separated from the same coal thus indicating different chemical environment for the free radical species and, possibly, presence of different radicals in some samples [2]. The Mark I spectrometer had somewhat limited capabilities; in particular a relatively narrow magnetic field scan range and variable temperature only down to $150 \mathrm{~K}$. Furthermore, the probehead did not have a remote tuning arrangement, complicating operation and and increasing the time needed for certain of the experiments.

At the same time, emerging interest in the use of EMR in studies of a wider range of centers such as transition metal ions (which are also common impurities in carbonaceous materials) at high magnetic fields demanded a new spectrometer capable of:

- broad range of sample temperatures from $36.6^{\circ} \mathrm{C}$ down to $4 \mathrm{~K}$;

- broad (several Tesla) but still accurate (milli- and sub-milliTesla) magnetic field scan;

- quantitative rotation of small (submillimeter) crystals; and

- capability for samples exhibiting long relaxation times (those are very typical for coal samples)

that usually result in rapid-passage EMR spectra;

- stability in magnetic field scans and resonance frequency to at least a ppm level.

To meet these challenges, we built a new W-band instrument (Mark II) that has been frequently upgraded since it became fully operational in 1995.

\section{MEASUREMENTS}

\section{The Mark II W-band (95 GHz) EMR spectrometer.}

The new Mark II W-band EMR spectrometer we built outperforms its prototype in many, though not all, respects. Briefly, Mark II features:

- an Oxford custom-built $7 \mathrm{~T}$ superconducting magnet which is scannable from 0 to $7 \mathrm{~T}$ at up to $0.5 \mathrm{~T} / \mathrm{min}$;

- water-cooled coaxial solenoid designed and built at the University of Illinois with up to $\pm 550 \mathrm{G}$ scan under digital (15 bits resolution) computer control; custom-engineered precision feed-back circuit based on an Ultrastab 860R sensor that has linearity better than $5 \mathrm{ppm}$ and resolution of $0.05 \mathrm{ppm}$;

- an Oxford CF 1200 cryostat modified at the University of Illinois to accommodate a new $\mathrm{W}$-band probehead with remote tuning and one-axis goniometer for vertical bore solenoid.

This spectrometer is operated on a daily basis and is also available to users who 
Semiannual Technical Report to DOE - PETC - Grant \# DE-FG22-96PC96205 9/01/97-2/28/98

collaborate with this research group. Some technical details and applications which are beyond the scope of this report are provided in our recent publications [3-8].

\section{Recent upgrades of the W-band (95 GHz) EMR spectrometer.}

During the last year we have carried out several important upgrades to keep up with the challenges set up by the applications. Particularly we have:

- replaced the entire waveguide of the low temperature probehead with a flexible dielectric Goretex waveguide section; this upgrade provides superb cavity temperature insulation important for cryogenic experiments with carbonaceous materials and metalloproteins, low microwave loss, and vacuum-tightness; thus, we no longer need a vacuum window on the top of cryostat which caused microwave losses, reflections, and phase shift of the signal; this waveguide is also an attractive solution for $\lambda$-point EPR experiments;

- incorporated a low-noise $95 \mathrm{GHz}$ amplifier (Millitech) which has $21.8 \mathrm{~dB}$ gain and 4.8 $\mathrm{dB}$ noise figure at $94.3 \mathrm{GHz}$; use of this amplifier in many cases eliminates rapid-passage conditions in cryogenic EPR experiments thus improving spectral resolution. (Long relaxation times and rapid passage conditions are typical for many organic free radicals and particularly coals at cryogenic temperatures.);

- developed a high Q ( $\approx 4000)$ cylindrical resonator which provides high concentrational sensitivity for aqueous samples and slurries of carbonaceous materials comparable to that of traditional X-band EMR.

In addition, we have carried out extensive tests of the Oxford magnet in order to characterize the hysteresis-like behavior during wide (several Teslas) scans [9]. NMR and Halleffect Gausmeters as well as a high-speed current transducer have been used for tracking the magnetic field and current through the solenoid and their accuracy have been compared, in order to measure the field as accurately as possible. The non-linear hysteresis-like behavior was found to be repeatable, thus allowing the use of the current-feedback as one of the mechanisms in controlling magnetic field in experiments requiring several Tesla scans, such as metalloproteins' problems often require. The Mark I system, which provides an exceptionally stable field and convenient 300-Gauss sweep, was restored to full operation and outfitted with better computer interfacing and control as well as with a fast switch for pulsed operation.

\section{RESULTS, DISCUSSION, and CONCLUSIONS}

In this report we focus on fusinite to demonstrate the power of HF EMR method in studies of carbonaceous solids. Fusinite was chosen because it is one of the most homogeneous fractions on natural coals known and has a low level of inorganic impurities. In particular, we studied the effect of oxygen on the fusinite EPR spectrum. One reason for this focus is that, based on our recent magnetic susceptibility measurements, we have already speculated that oxygen might play a substantial role in spin-spin interactions in fusinite and other carbonaceous solids [10]. A second and also important reason is that fusinite is now used as a probe to measure oxygen [11] and nitric oxide [12] concentration in vivo and in vitro and detailed understanding of 
Semiannual Technical Report to DOE - PETC - Grant \# DE-FG22-96PC96205 9/01/97-2/28/98

oxygen effects on EMR spectrum are important for synthesizing new carbonaceous EMR probes for simelar purposes.

The conventional EMR X-band spectrum of fusinite is a single featureless line with the width responding to the oxygen concentration. An example of such a spectrum for a carefully deoxygenated sample is shown in Fig.1 (A). It is clear that the spectrum has broad wings which cannot be modeled by a Lorentzian function or inhomogeneous line shape model. These wings could due to presence of several components with different $T_{2}$ but almost identical $g$-factor or to peculiarities of spin- spin interactions. For example, incompletely averaged dipole-dipole interaction is known to cause some extra broadening. In a theoretical study, Salikhov and coworkers have calculated the free induction decay (FID) for a system of $S=1 / 2$ spins with anisotropic g-factors interacting via dipolar coupling [13]. A model of magnetically diluted solids in which dipolar broadening is much less than inhomogeneous line width, thus allowing an adiabatic approximation for the dipole-dipole interaction, was considered. It has been shown that in the high temperature limit, when dipole-dipole interaction is modulated by random spin flipflops, the spin polarization effects are negligible, and the FID, $v_{\mathrm{FID}}(\mathrm{t})$, is no longer a simple exponential but rather takes the form:

$$
\mathrm{U}_{F I D}(t) \approx \exp \left(-a(t)^{1 / 2}\right), \quad[1]
$$

where:

$$
a=\frac{8 \pi^{3 / 2}}{3^{5 / 2}}\left\langle g^{2}\right\rangle \beta^{2} \hbar^{-1} C\left(T_{1}\right)^{1 / 2},
$$

$\left\langle\mathrm{g}^{2}\right\rangle$ is a weighted $\mathrm{g}$-factor for the two interacting species [13], C is spin concentration, and $T_{1}$ is the spin-lattice relaxation time. The corresponding $\mathrm{CW}$ spectrum $\mathrm{V}(\omega)$ is given by a Fourier transform and is not Lorentzian; while the central part is slightly sharper than the Lorentzian for the same peak-to-peak line width $\triangle \mathrm{B}_{\mathrm{p}-\mathrm{p}}$, the wings vanish less rapidly. As a result, dipolar broadening as given by eq. [1] decreases the peak-to-peak intensity of the $\mathrm{CW}$ spin-label spectrum by adding very broad wings with only a little broadening of the central part.

We used this model to fit the experimental spectrum using a fast convolution algorithm based on Levenberg-Marquardt optimization method [14]. In Fig.1, the fusinite X-band EMR spectrum is superimposed with least-squares simulations that prove to match closely the experiment. The residual of the fit, i.e. a difference between the experimental and the simualted spectrum in shown in Fig. 1 B.

This exceptional fit demonstrates that the fusinite spectrum could be well described by a model of a single-type paramagnetic species which EMR line shape that is determined by spin 
Semiannual Technical Report to DOE - PETC - Grant \# DE-FG22-96PC96205 9/01/97-2/28/98

exchange and dipole-dipole interaction. Under deoxygenation, the peak-to-peak fusinite line width initially decreases very rapidly with some further small changes observed over a period of a few hours (Fig. 2). The fast phase of deoxygenation is likely related to molecular oxygen been removed from the fusinite porous structure while the slow phase could be related to a slower process determined by the oxygen diffusion in the carbon matrix.

This slow process which is barely measurable from X-band spectra looks very different at W-band (Fig 3): in half an hour the line first becomes slightly distorted and then two components arise, change widths, and separate further. This two components can be well modeled and separated using the same dipolar fitting model we developed for the X-band fusinite spectrum (Fig. 4).

One could say that X-and W-band experiments contradict each other: while the X-band spectrum is narrowing in the course of a continuous deoxygenation, the $\mathrm{W}$-band spectrum (overall peak-to-peak line width measured as distance between maximum and minimum) is actually broadening. However, this puzzling behaviour can be explained in terms of exchange theory and our hypothesis that molecular oxygen could serve as a bridge for those interactions. The line width of fusinite at X-band is primarily determined by both the spin exchange and the dipoledipole interactions between the paramagnetic species in solid; the oxygen serves as an additional relaxer for these species and it causes some extra broadening also by the exchange mechanism. Complete deoxygenation does not break the existing exchange conditions (exchange frequency is higher than $9.5 \mathrm{GHz}$ ) so the spectrum remains unresolved while the width gradually decreases while oxygen is removed from solid matrix and absorption sites.

At W-band, the exchange frequency becomes lower than the frequency of the experiment as oxygen is removed from the system. We speculate that oxygen serves as a "bridge" for spin exchange; removing the oxygen thus decreases the effective exchange frequency. This causes the signals to split and to separate further in course of experiment.

Additional support of this exchange condition can be deduced from multifrequency EPR data given in [2]. Those data, taken at the very initial stage of deoxygenation, show that the line width actually drops a little from 1 to $9.5 \mathrm{GHz}$ and then increases as the EMR frequency increases. This decrease in the line width is in accord with Anderson-Weiss theory, which predicts a decrease in the peak-to-peak line width for exchange-narrowed lines. At higher frequencies, the differences in $\mathrm{g}$-factors cause an initial broadening which is proportional to $(\triangle \mathrm{g} \cdot \mathrm{B})^{2}$ before lines begin to split.

\section{REFERENCES}

[1] Wang, W., Belford, R. L., Clarkson, R. B., Davis, P. H., Forrer, J., Nilges, M. J., Timken, M. D., Walczak, T., Thurnauer, M. C., Norris, J. R., Morris, A., L., and Zwang, Y., 1994, Very High Frequency EPR - $94 \mathrm{GHz}$ Instrument and Applications to Primary Reaction Centers from Photosynthetic Red Bacteria and to Other Disordered Systems, Appl. Magn. Reson. 6: 195-215.

[2] Clarkson, , R. B., Wang, W., Brown, D. R., Crookham, H. C., and Belford, R. L. Electron Magnetic Resonance of Standard Coal Samples at Multiple Microwave Frequencies In 
Semiannual Technical Report to DOE - PETC - Grant \# DE-FG22-96PC96205 9/01/97-2/28/98

Magnetic Resonance of Carbonaceous Solids (Botto, R. E., and Sanada, Y. Eds.) Advances in Chemistry Series 229, American Chemical Society, Washington, DC 1993, pp. 507-528.

[3] Dalal, N. S., Smirnov, A. I., Smirnova, T. I., Belford R. L., Katritzky, A. R., and Belyakov, S. A. "Single Crystal Multi-Frequency EPR Evidence for a Quasy Low-Dimensional Spin Exchange in 3-n-butyl-2,4,6-triphenylverdazyl", J. Phys. Chem., 1997.

[4] Dalal, N. S., Smirnov, A. I., and Belford R. L. "Quantification of Coal-Diesel Particulate Mixtures by W-band (94 GHz) ESR Spectroscopy", accepted, Appl. Spectr. 1997.

[5] Hustedt, E. J., Smirnov, A. I., Laub, C. F., Cobb, C. E., and Beth, A. H. "Measurement of Molecular Distances Using Dipolar Coupled Nitroxide Spin-Labels: The Global Analysis of Multifrequency CW-EPR Data", Biophys. J., 74, 1861-1877 (1997).

[6] Smirnova, T. I., Smirnov, A. I., Clarkson, R. B., Belford, R. L., Kotake, Y., and Janzen, E. G. "High-Frequency (95 GHz) EPR Spectroscopy to Characterize Spin Adducts", J. Phys. Chem. $B, \mathbf{1 0 1}, 3877-3885$ (1997).

[7] Britton, M. M., Fawthrop, D. G., Gillies, D. G., Sutcliffe, L. H., Wu, X., and Smirnov, A. I. "Matched Spin Probes for the Study of the Overall Motion of Model Lubricants", Magn. Reson. Chem. 35, 493-502 (1997).

[8] Smirnova, T. I., Smirnov, A. I., Belford, R. L., and Clarkson, R. B. "Interaction of MRI Gadolinium Contrast Agents with Phospholipid Bilayers as Studied by $95 \mathrm{GHz}$ EPR", Acta Chemica Scandinavica, 51, pp. 562-566 (1997).

[9] Perkins, R. C., J r., M unro, N., Dierker, J., M orse, P. D., II, Smirnov, A. I., Nilges, M. J.,Clarkson, R. B, and Belford, R. L., "Characterization and Control of Superconducting Magnets Suitable for EM R Spectroscopy of Transition M etals at F requencies $>90 \mathrm{GHz}$ ", 39th Rocky M ountain Conference on A nalytical Chemistry, Denver, CO, A ugust (1997).

[10] Smirnova T. I., Smirnov A. I., Clarkson R. B., and Belford R. L., "Magnetic Susceptibility and Spin Exchange in Fusinite and Carbohydrate Chars", J. Phys. Chem., 98 (10), pp.2464-2468, (1994).

[11] Vahidi, N., Clarkson, R. B., Liu, K. J., Norby, S. W., and Swartz, H. M. In Vivo and In Vitro EPR Oximetry with Fusinite: a New Coal-Derived Particulate EPR Probe. Magn. Reson. Med. 31, 139-146 (1994).

[12]Clarkson R. B., Norby S. W., Smirnov A. I., Vahidi N., Nims R. W., and Wink D. A., "Direct Measurement of the Accumulation and Mitochondrial Consumption of Nitric Oxide Within Chinese Hamster Ovary Cells Using a Novel Intracellular Electron Paramagnetic Resonance Technique", Bioch. Bioph. Acta, 1243, pp. 496-502, (1995).

[13] Maryasov, A. G.; Dzuba, S. A.; and Salikhov, K. M. J. Magn. Reson. 1982, 50, 432450.

[14] Smirnov, A. I., and Belford, R. L., "Rapid Quantitation from Inhomogeneously Broadened EPR Spectra by a Fast Convolution Algorithm", J. Magn. Reson. A, 98, pp. 65-73, (1995) 
Semiannual Technical Report to DOE - PETC - Grant \# DE-FG22-96PC96205 9/01/97-2/28/98

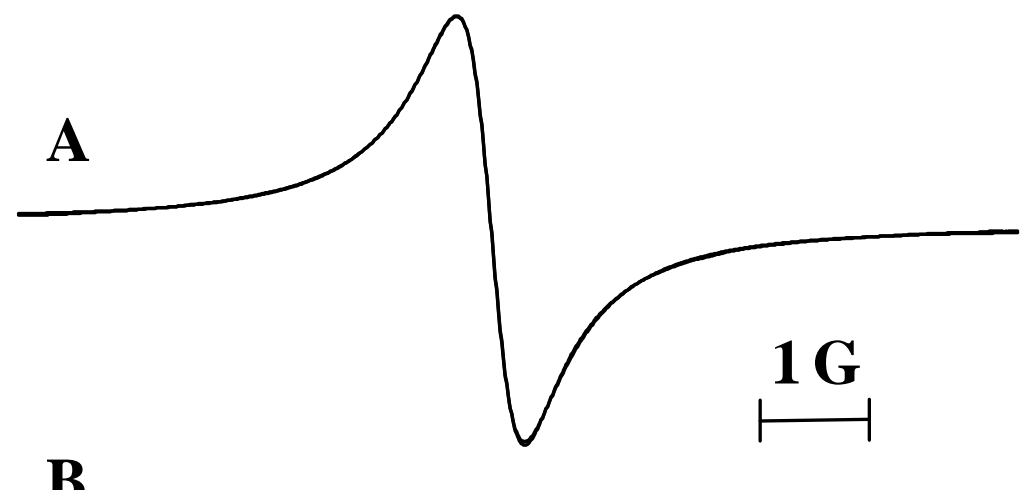

Figure 1. Experimental 9.5 GHz (X-band) EMR spectrum of deoxygenated fusinite sample and the results of least-squares simulations based on high-temperature-limit dipolar model (A), (B) is the fit residual.

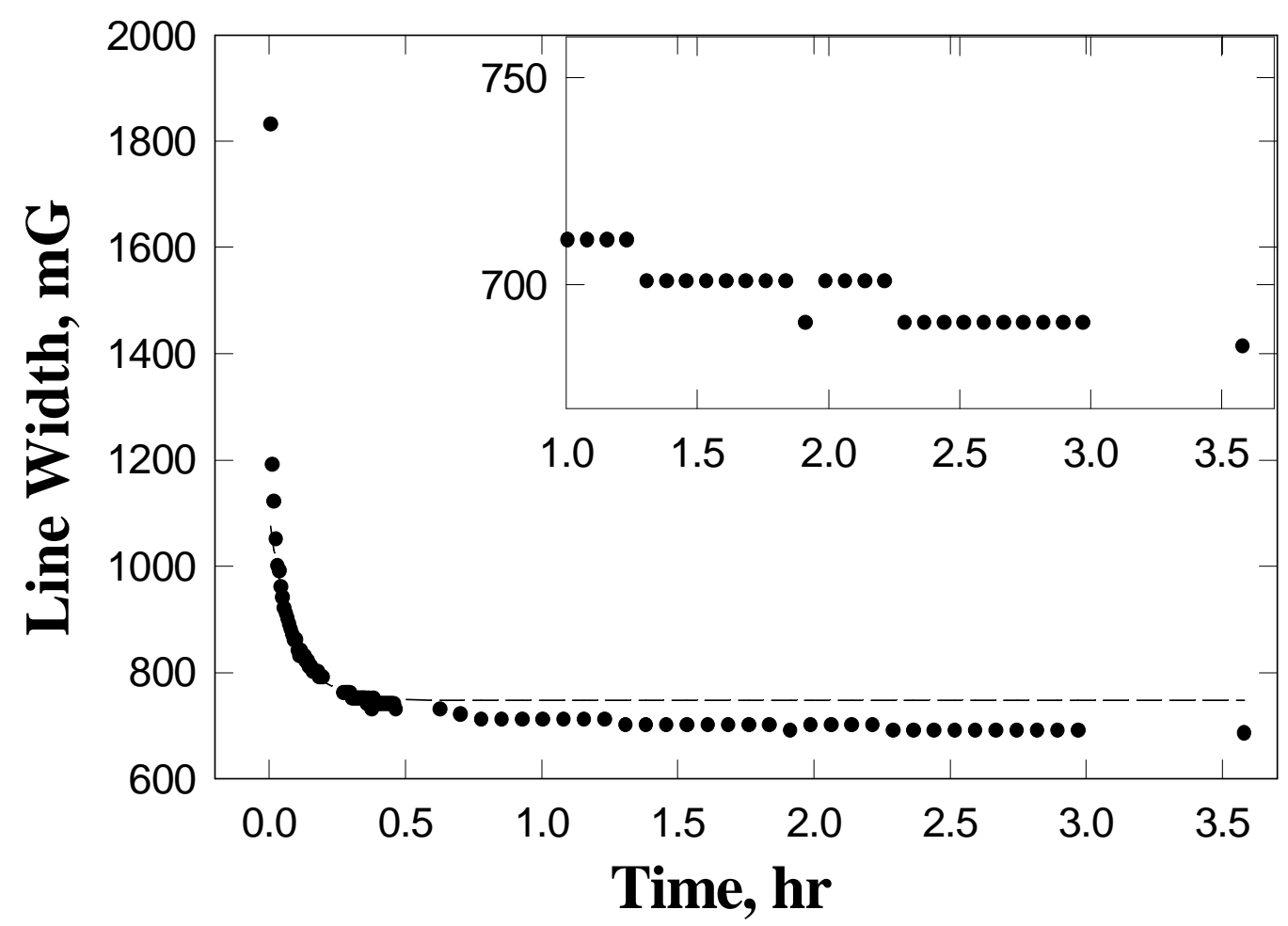

Figure 2. Kinetics of apparent peak-to-peak line width of the fusinite sample in course of continuous deoxygenation as measured at X-band. 
Semiannual Technical Report to DOE - PETC - Grant \# DE-FG22-96PC96205 9/01/97-2/28/98

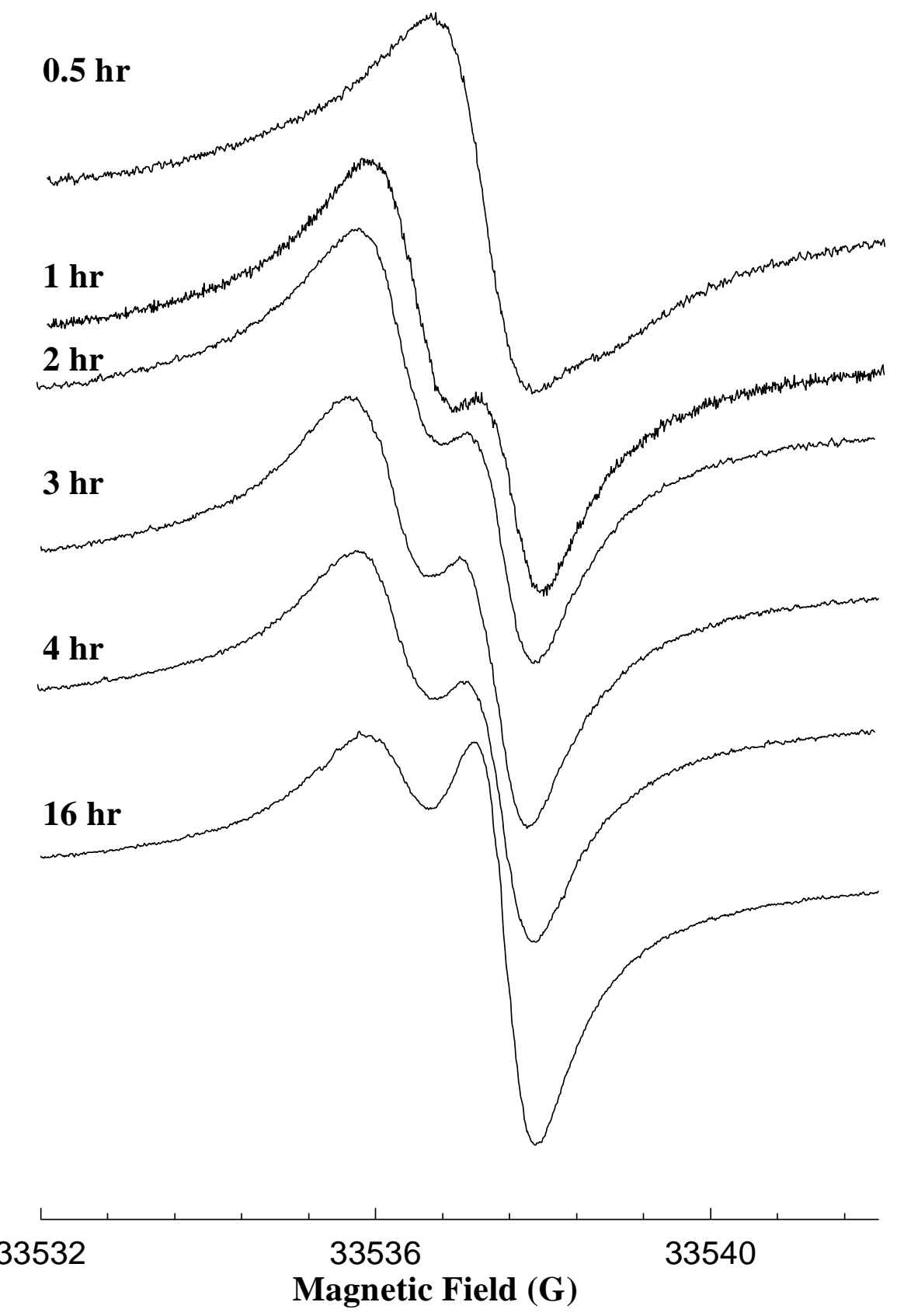

Figure 3. Gradual changes in $95 \mathrm{GHz}$ EMR spectra of fusinite in course of deoxygenation 


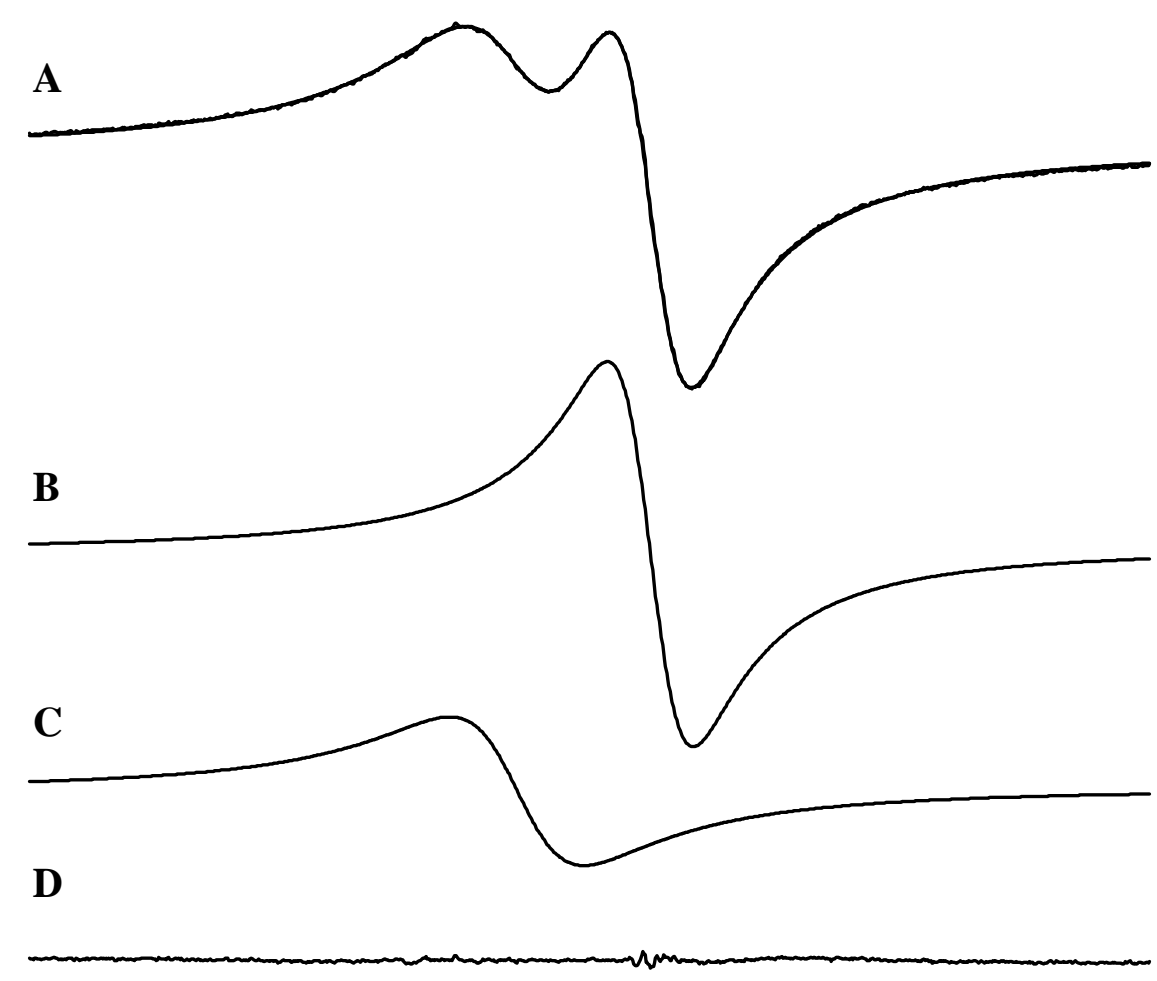

\section{$\begin{array}{lllll}33534 & 33536 & 33538 & 33540 & 33542\end{array}$ Magnetic Field, G}

Figure 4. Experimental $95 \mathrm{GHz}$ (X-band) EMR spectrum of fusinite sample deoxygenated for 16 hrs is superimposed with the results of least-squares simulations; (B) is the simulation of first component; (C) is the second component, and (D) is the fit residual. 